\title{
Esophageal Choke and its Management in a Thorough Bred Horse
}

\author{
Adolfo F Godoy Pinto* \\ Department of Clinical Sciences, University Of Chile, South Africa
}

Received: July 10, 2017; Published: July 14, 2017

*Corresponding author: Adolfo F Godoy Pinto, Department Of Clinical Sciences, Area Of Internal Medicine And Equine Surgery, Faculty Of Veterinary Sciences, University Of Chile, South Africa, Email: agodoy@uchile.cl

\begin{abstract}
We describe the clinic case of a thoroughbred horse with esophageal obstruction that presented a fatal outcome, possibly attributed to inadequate management in which excessive use of the nasogastric tube to push the contents was responsible of ulceration and inflammation of the esophageal mucosa with posterior cicatricial reaction and irreversible stenosis of the organ. The importance of medical therapy, based on analgesics, anti-inflammatory, sedatives (alpha 2 agonists), oxytocin, is highlighted. In addition, the use of nasogastric tube only to dissolves the obstructive content using soft pressure water and not to push content.
\end{abstract}

Keywords: Thoroughbred horses; Dysphagia; Esophagus; Choke; Oxytocin

\section{Introduction}

The esophagus has mucosal, sub mucosal, muscular, and adventitial layers. A serosal layer is present for the very short segment of the esophagus that traverses the abdominal cavity between the diaphragm and the stomach. The proximal two thirds of the esophagus contain skeletal muscle, whereas the distal third of the esophagus is composed of smooth muscle [1].

Esophageal obstruction, either primary (simple choke), or secondary to other disease processes is the most common esophageal disorder seen in horses. The cause of primary obstructions are many, however roughage in the diet and stall bedding materials are frequently identified. Prior esophageal trauma or poor mastication caused by dental abnormalities may predispose horses to esophageal impaction $[1,2]$.

However, in the author's experience in thoroughbred horses, the main predisposing factors are; animals fed after strenuous post competition exercise or those who have undergone general anesthesia. In these two circumstances there are common factors; in both cases are animals that have been subjected in a greater or lesser degree to a period of fasting, in the same way, they also have some degree of dehydration, all this results in a fast ingestion of the food when it's offered to the animal, forming in its mouth a dry bolus, which increases the possibility of an esophageal obstruction, especially considering that the main type of food that receives is dry grass and pellet.
Although it's true that a significant number of these cases are self-limited, some can be transformed into serious pathologies, such as the occurrence of aspiration pneumonia, esophageal rupture, or a severe cicatricial reaction of the esophagus mucosa [3], in this last case the mucosa is replaced by fibrous tissue narrowing the lumen and preventing the normal transit through the organ [4], both situations can have a fatal outcome.

\section{Clinical Case}

A 6-year-old horse had been treated by his veterinarian for five days due to esophageal obstruction, according to anamnestic information; the patient was treated with analgesics, antiinflammatory, sedatives, oxytocin, in therapeutic doses and mechanical decompression with nasogastric tube. Because of this, the removal of the foreign body from the esophagus was achieved. Twenty-four hours after treatment the horse received water and food in small amounts, however at 72 hours the animal had recurrence of clinical signs, then the veterinarian repeated the same treatment scheme described above several times, using the nasogastric tube, with no results positive, so he decided to move the horse to the teaching hospital of the University. When the patient was received in the teaching hospital he showed depression (in the interpretation of this sign should be considered the repeated medication with alpha 2 agonists performed by his veterinarian), the horse was standing with the neck extended, bilateral nasal foam flow which contains saliva and food material, cough, odynophagia, ptyalism and dysphagia with repeated attempts to swallow. 
Palpation of the jugular furrow revealed a mass associated with the impaction at the first third of the esophagus. Heart rate was 50 beats / minute, and a respiratory rate of 25 breaths / minute, auscultation of the lungs was normal, capillary refill time of three seconds, skin tent of five seconds, mucous membranes congestive. Hemogram with the classic reaction of stress (leukocytosis with neutrophilia and lymphopenia) and biochemical profile within normal ranges. In these values should be considered the medication to which his veterinarian has subjected the animal and that could distort the observation made. Radiographic (Figure 1) and Ultrasonography evaluation of the cervical region confirmed the presence of a cervical esophageal impaction in the first third of the esophagus, which was corroborated by endoscopy (Figure 2).

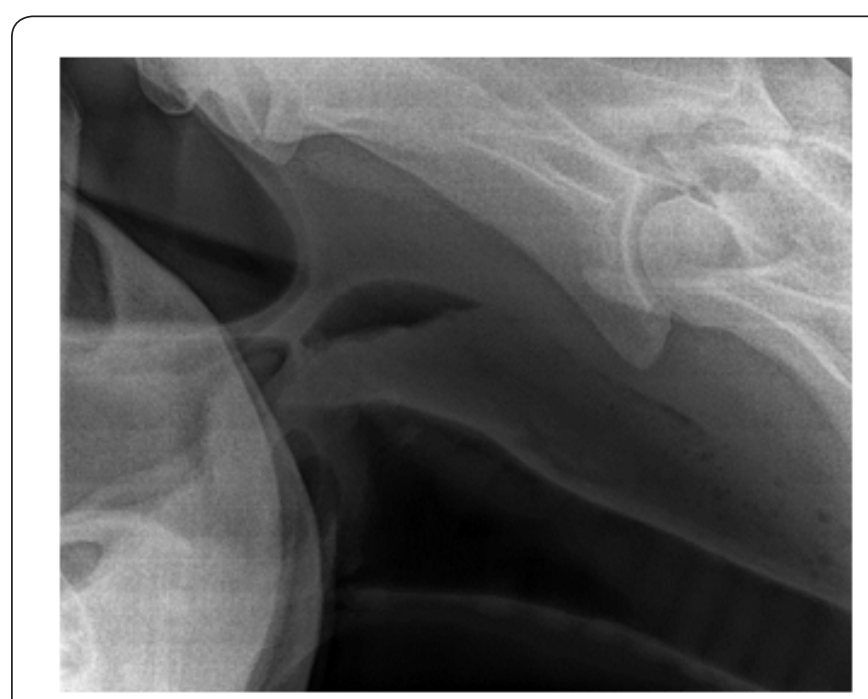

Figure 1: Radiographic image showing dilatation of the esophagus by contents inside.

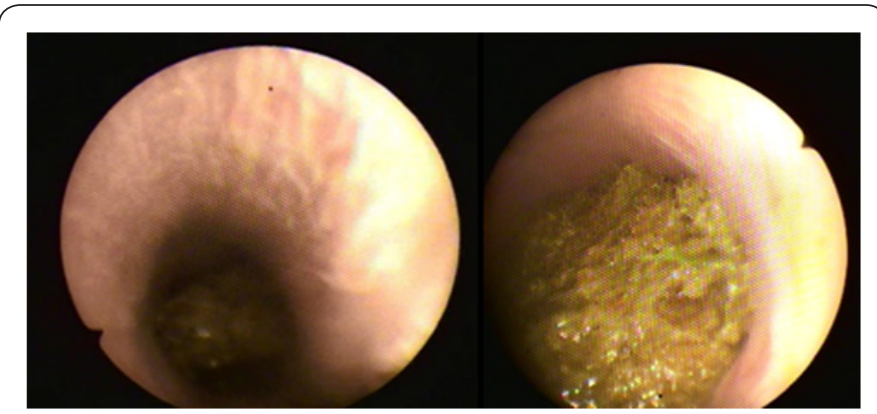

Figure 2: Endoscopic appearance of fibrousfeed impaction of the esophagus.

\section{Treatment}

Fluid therapy was performed considering anestimate dehydration at 5\%; oxytocin at a rate of $0.1 \mathrm{IU} / \mathrm{kg}$ body weight [5] was given in $500 \mathrm{ml}$ of saline solution twice in a 30 -minute interval [6]. Then nasogastric tube was placed until the obstruction and the content was then dissolved using warm water at gentle pressure keeping the head of the animal down [7]. Once the obstruction was resolved, the animal was calm, stopped the sialorrhea, the nasal reflux, and kept its neck and head in normal condition. The endoscopic assessment of the esophagus following resolution of the impaction is very important to determine whether mucosal ulceration, esophageal rupture, masses, diverticula or strictures are present, since this depends on the prognosis of the clinical case [1]. In this case, esophageal endoscopic examination showed a very ulcerated and inflamed mucosa at the site of the obstruction (Figure 3). The radiological evaluation of the esophageal with contrast medium barium showed a significant decrease in the esophageal lumen.

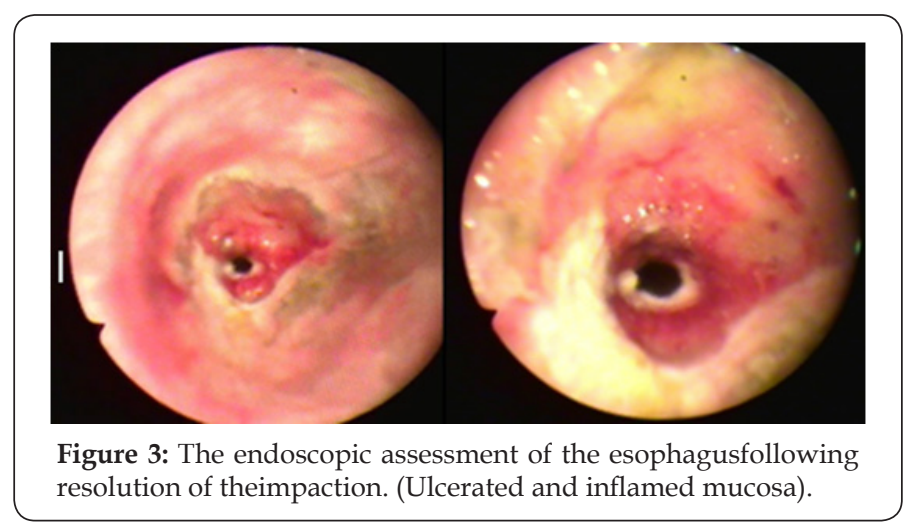

Parenteral feeding, antibiotics (combination of beta-lactams and aminoglycosides) and corticosteroids in therapeutic doses were given for 6 days; an endoscopic evaluation of the esophagus was performed every 48 hours, observing a progressive stenosis (strictures) of the site where the obstruction was. On the seventh day post-treatment, the esophagus showed occlusion greater than $90 \%$ of its normal lumen and the radiography with contrast medium barium showed the impossibility of passage of liquid through the organ. Considering the critical diagnosis of the case, and based on the poor prognostic that could have an esophageal surgery in these conditions, the owner and his veterinarian decided to carry out the euthanasia of the animal

\section{Conclusion}

Although the esophageal obstruction, as mentioned above, is in many cases self-limiting, the prognosis will be determined by the type of obstructive material, the time it takes the problem to be solved and the appropriate medical management to be made of the case. In this case, in the opinion of the author, although the correct medications were administered, it was not appropriate to prolong the time needed to solve the problem and the repeated attempt to resolve the obstruction by mechanical use of the nasogastric tube; this caused severe mucosal damage leading to stenosis of the esophageal lumen.

The use of nasogastric tube in the procedure should only be for detect the site of obstruction and then proceed to dissolution using warm water under gentle pressure, never use the nasogastric tube to push the material since the risk of producing damage leading to fibrosis and stenosis irreversible scarring in the esophagus is very high. In the author's experience, the surgical alternative of esophageal obstruction has a poor prognosis, due to secondary esophageal strictures after surgery and the absence of serosa in the first two thirds of the organ favors dehiscence of the suture after the surgery. 


\section{References}

1. Mathew PG (2007) Esophageal choke and its management. Proceeding of the NAVC North American Veterinary Conference, Orlando, Florida.

2. Brazil T (2011) How to approach the horse with choke. Proceedings of the 50th British Equine Veterinary Association Congress BEVA Liverpool, United Kingdom. pp: 249-250.

3. Craig DR, Shivy DR, Pankowski RL, Erb HN (1989) Esophageal disorders in 61 horses. Results of nonsurgical and surgical management. Vet Surg 18(6): 432-438.

4. Jeldborg J, Keith EB (2011) Diagnosis and Treatment of Dysphagia. 12th International Congress of World Equine Veterinary Association (WEVA), Hyderabad, India.
5. Meyer G, Helms R, Rashmir-Raven A, Brashier M (2000) Effect of Oxytocin on Contractility of the Equine Esophagus: Treatment for Esophageal Obstruction. Proceedings of the Annual Convention of the AAEP 32(2): 151-155.

6. Godoy (2009) Obstrucción esofágica en equinos (“Choke”) Revista Tecnovet. 15(1): 17-22.

7. Hance S, Noble J, Holcomb S (1997) Treating Choke with Oxytocin. Proceedings of the Annual Convention of the AAEP 43: 338-339.

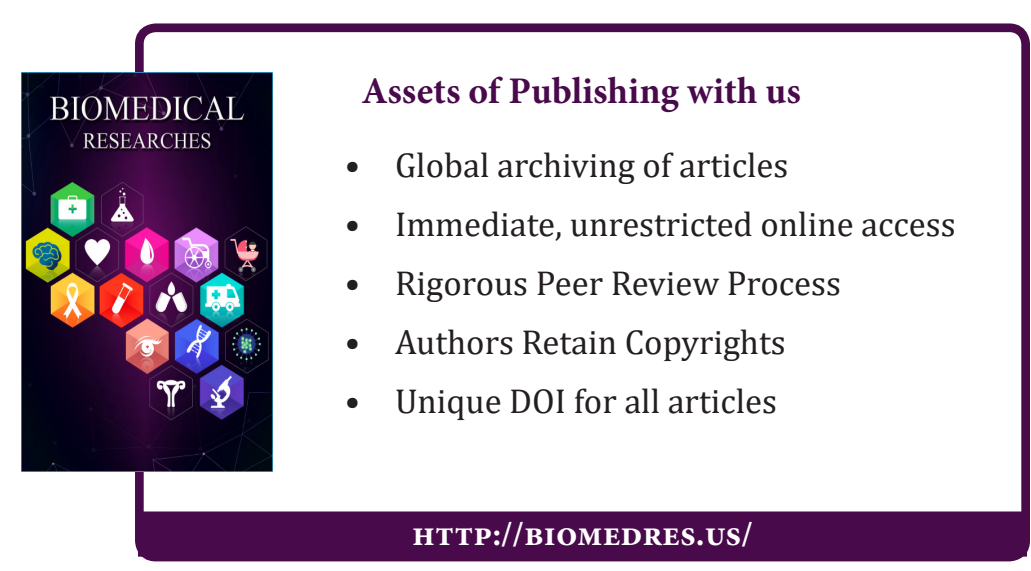

